\title{
Adaptive optics for control of the laser welding process
}

\author{
Libor Mrňa $^{\mathrm{a}}$, Martin Šarbort, Šimon Řeřucha, and Petr Jedlička \\ Institute of Scientific Instruments, AS CR, Královopolská 147, Brno, 612 64, Czech Republic
}

\begin{abstract}
The laser head with fixed focus optics is commonly used for the deep penetration laser welding. In such case the geometry and position of the beam waist are defined by the focusing lens. If the laser beam incident on the focusing lens is not well collimated but divergent and its divergence can be varied by proper adaptive optical elements, then also the geometry and position of the focus will be changeable. In this way it is possible to affect the energy coupling from the laser beam to the keyhole walls and thus to control the geometry and quality of the weld. In this paper we present a theoretical and numerical study of the beam shaping by adaptive optics and its influence on the weld quality. For the $\mathrm{CO}_{2}$ laser welding machine the adaptive optics was realized by a deformable mirror and its effect was tested also experimentally. For the solid-state laser welding machine we designed a laser head with changeable distance between the optical fiber and the collimating lens and we simulated its performance.
\end{abstract}

\section{Introduction}

A principle of deep penetration laser welding lies in interaction of high power laser beam with welded material. A thin capillary called keyhole is created in the material in the case of sufficient intensity incident on material joint. Its depth is almost identical to the depth of the resulting weld. The properties and behavior of the keyhole during the welding process depend on welding parameters among which the most important are thermo-mechanical characteristics of the welded material, welding speed, laser beam power and its spatial shape described by divergence, waist size and focus position with the respect of surface of the work piece.

Geometric parameters of the laser beam significantly affect the propagation of the energy radiation within the keyhole, where it undergoes multiple reflections and gradual absorption at the keyhole walls, and consequently influence a spatial distribution of the total absorbed intensity. For the amount of absorbed intensity is strongly related to other physical quantities that play a role in the welding process, e.g. temperature distribution in the molten material or surface temperature of the keyhole walls, it is necessary to achieve its optimal distribution. For given power and welding speed it might be done by optimization of laser beam focus characteristics. On the contrary an inappropriate choice of these parameters can lead to dramatic decrease of penetration and quality of the weld.

Several papers that analyze the dependence of keyhole characteristics on the geometric parameters of the laser beam have been published during the last years. The absorbed intensity distribution within the keyhole was studied in [1-4]. The dependence of the keyhole depth on the waist size and its position with respect to the work piece was discussed in $[5,6]$. Complex simulations of keyhole creation involving the multiple reflections were published in [7-12].

\footnotetext{
a e-mail:mrna@isibrno.cz
}

This paper presents a study of a correspondence between geometric parameters of the laser beam (divergence, waist size, focus position) and the keyhole characteristics which was motivated by an extremely wide range of possible settings of laser beam parameters enabled by our welding machine. The capability to adjust the laser beam parameters was achieved by implementation of a special active element into the optical system of the welding machine. Actually, the active element is realized by a mirror with a variable curvature referred to as an adaptive mirror. This mirror enables us to actively shape the laser beam and consequently influence the keyhole characteristics.

For the purpose of the study of the relations between the keyhole properties and laser beam geometric shape a two-dimensional physical model of the keyhole creation has been evolved and on the basis of this model a numerical simulation has been implemented. Although not all possibly participating physical phenomena were included, the numerical simulation gives relevant information about energy density and surface temperature distribution at the front and rear wall of the keyhole in dependence on laser beam geometric parameters. The keyhole depths obtained for various beam shapes from numerical simulation can also be compared to the depth of real material joint observed in material samples from testing welds.

\section{Laser beam propagation in optical system}

This section describes an experimental arrangement of the optical system of our welding machines and a method applicable for the calculation of the beam geometry after its propagation through this system.

\section{$2.1 \mathrm{CO}_{2}$ laser welding machine}

The focusing system of our $\mathrm{CO}_{2}$ welding machine is illustrated in Figure 1. The laser beam outgoing from an initial

This is an Open Access article distributed under the terms of the Creative Commons Attribution License 2.0, which permits unrestricted use, distribution, and reproduction in any medium, provided the original work is properly cited. 


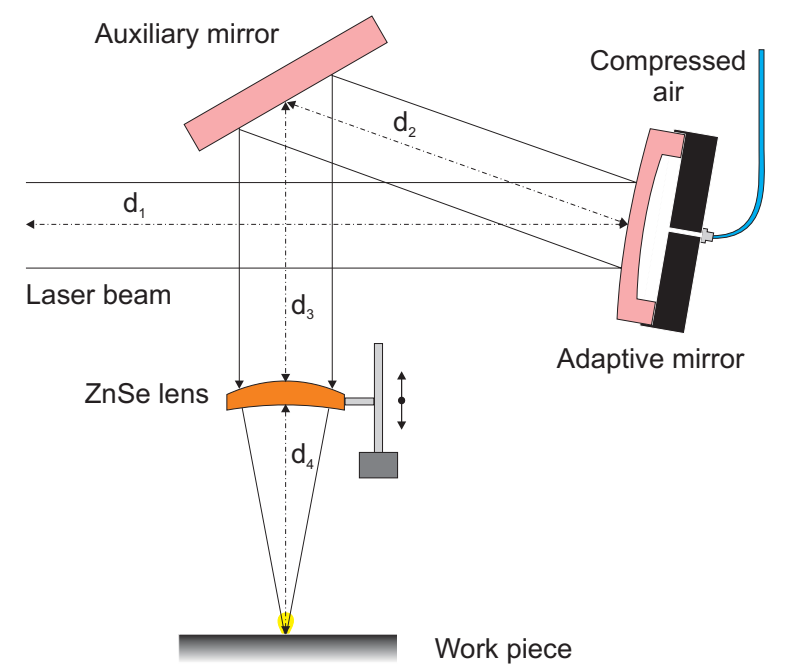

Fig. 1. Optical system of the $\mathrm{CO}_{2}$ laser welding machine

waist (its position is given by laser source) is gradually reflected by the adaptive mirror and an auxiliary plain mirror, after that it is focused by the converging lens of focal length $f$ to the final waist of radius $w_{0 \mathrm{f}}$. Changing either a curvature of adaptive mirror or vertical position or focal length of the lens we can change the position and radius of the final waist (inevitably, the beam divergence is also changed). For further analysis of the relations between the beam shape and keyhole characteristics, it is necessary to calculate the dependence of final waist geometry on the quantities describing the optical system.

This dependence can be calculated by a method called ray transfer matrix analysis [13]. Assuming the laser beam of wavelength $\lambda$ outgoing from the initial waist to be Gaussian, its shape along the beam axis $z$ can be described by a complex beam parameter $q(z)$ defined in general as

$$
\frac{1}{q(z)}=\frac{1}{z+\mathrm{i} z_{\mathrm{r}}}=\frac{1}{R_{\mathrm{w}}(z)}-\frac{\mathrm{i} \lambda}{\pi w(z)},
$$

where the coordinate $z$ is measured from the beam waist of radius $w_{0}$, function $w(z)$ describes the beam radius along the $z$-axis, $z_{\mathrm{r}}=\pi w_{0}^{2} / \lambda$ is Rayleigh range and $R_{\mathrm{w}}(z)$ is the radius of curvature of the wave fronts.

Given the initial beam parameter $q_{\mathrm{i}}$ and a ray transfer matrix $M=\left(\begin{array}{ll}A & B \\ C & D\end{array}\right)$ of the optical system, the resulting beam parameter $q_{\mathrm{f}}$ after passing the optical system is expressed by

$$
\frac{1}{q_{\mathrm{f}}}=\frac{C+D / q_{\mathrm{i}}}{A+B / q_{\mathrm{i}}}
$$

From the value of beam parameter $q_{\mathrm{f}}$ the position and radius $w_{0 \mathrm{f}}$ of the final beam waist can be expressed thanks to the simple condition - the value of $q_{\mathrm{f}}$ must be purely imaginary at the waist (the wave front is planar here).

The matrix $M$ of our optical system where the adaptive mirror is approximated by a spherical mirror of constant curvature radius $\rho_{\mathrm{m}}$ is given by a matrix product

$$
M=\left(\begin{array}{cc}
1 & d_{4} \\
0 & 1
\end{array}\right)\left(\begin{array}{cc}
1 & 0 \\
-1 / f & 1
\end{array}\right)\left(\begin{array}{cc}
1 & d_{3}+d_{2} \\
0 & 1
\end{array}\right)\left(\begin{array}{cc}
1 & 0 \\
-2 / \rho_{\mathrm{m}} & 1
\end{array}\right)\left(\begin{array}{cc}
1 & d_{1} \\
0 & 1
\end{array}\right),
$$
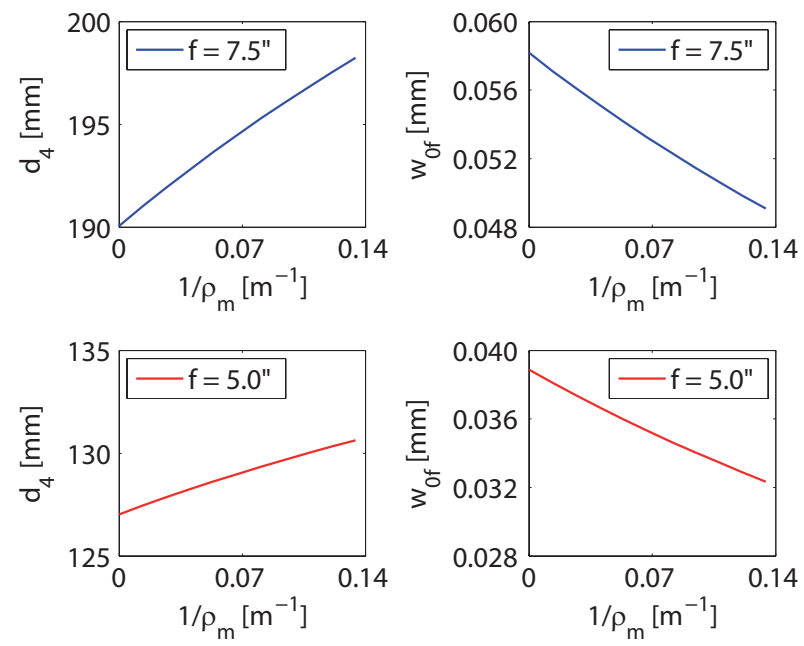

Fig. 2. Dependence of final waist radius $w_{0 \mathrm{f}}$ and its distance from converging lens $d_{4}$ on curvature of adaptive mirror $1 / \rho_{\mathrm{m}}$ for two different focal lengths $f$

where $d_{1}, d_{2}$ and $d_{3}$ are distances between particular optical elements, $d_{4}$ is a distance of the final beam waist from the converging lens (see Figure 1).

The resulting expressions for quantities $d_{4}$ and $w_{0 \mathrm{f}}$ depending on parameters describing the optical system are quite complicated to write them explicitly here, therefore we will rather present them in a graphical form. Figure 2 shows the most interesting dependence which is also the hardest to predict - the dependence of distance $d_{4}$ and radius $w_{0 \mathrm{f}}$ on the adaptive mirror curvature $1 / \rho_{\mathrm{m}}$ (with other optical system parameters fixed). It is apparent from the graphs that the distance $d_{4}$ increases as the curvature $1 / \rho_{\mathrm{m}}$ increases while the waist size $w_{0 \mathrm{f}}$ decreases as the curvature $1 / \rho_{\mathrm{m}}$ increases. Note that this dependence is inverse when the beam shape is changed by varying the focal length of the converging lens.

\subsection{Solid-state laser welding machine}

Another welding machine we employ for experiments is based on the solid-state laser (SSL) source. Due to the different working wavelength the laser beam is delivered to the focusing optics through an optical fibre and, therefore, the design of the welding head significantly differs from the welding head employed with $\mathrm{CO}_{2}$ welding machine. The main difference is apparent from Figure 3 - there is a collimating and a focusing lens but no adaptive mirror. Therefore we need another approach in order to achieve the possibility of changing the laser beam geometry.

The design of adaptive optics for SSL is based on possibility of changing the distance between the end of optical fibre and the collimating lens $d_{1}$. This allows to change the divergence of the beam incident on the focusing lens and, consequently, to change the geometrical parameters of the focus located at a distance $d_{3}$ from the focusing lens.

The beam parameters after passing through the optical system can be calculated by the same method of ray transfer matrix analysis described above. The dependence of the distance $d_{3}$ and corresponding waist radius $w_{0 \mathrm{f}}$ on the distance $d_{1}$ is shown in Figure 4. It is apparent that the dependence has similar character as the one obtained for the welding head with adaptive mirror. 
Optics and Measurement 2012

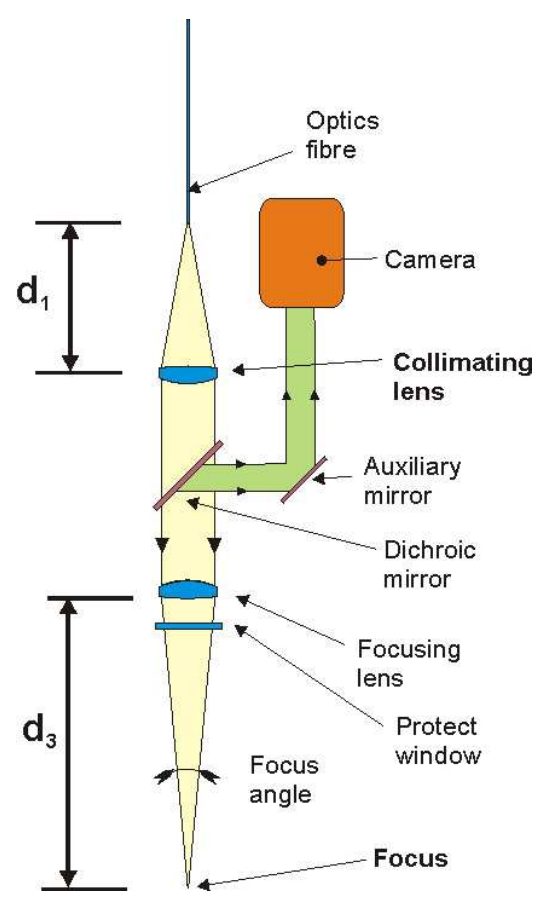

Fig. 3. Optical system of the solid-state laser welding machine

\section{Physical model of keyhole creation}

It follows from the results of previous section that the optical system parameters strongly affect the geometry of laser beam incident on the work piece. In order to study the consequences for keyhole properties a physical model of keyhole creation has been evolved. This section deals with general characteristics of this model and physical phenomena that are involved.

The physical model of the keyhole creation is based on the following simplified notion: The laser beam with defined intensity profile and spatial shape moves with constant welding speed $\mathrm{v}$ along the work piece. The energy radiation of the beam hits the surface of the work piece where it is partially absorbed. Some of the absorbed intensity is dissipated into the material due to the heat conduction while the rest causes a warming-up of the material. When a small volume of the material reaches the maximum temperature corresponding to the boiling point, it is removed from the system. An increasing total volume of removed material leads to the creation of the keyhole body. Within the emerging keyhole the laser beam undergoes multiple reflections on its walls which in turn increases the total amount of the absorbed intensity. On the other hand, the intensity of the beam becomes attenuated due to absorption in plasma that fills the keyhole and forms a plasma plume above it. As the laser beam moves along the work piece the molten material flows horizontally from the keyhole front wall to the rear wall.

Of course, this concept of keyhole creation process is very simplified. The idea of removing the material reaching the boiling point was accepted as a simplest way to replace the effects of phenomena which actually makes the keyhole creation possible. Among them the flow of the molten material caused by pressure gradient or evaporation of the material are the most important. However, as indicated further even such a simple model can provide relevant information about dependence of keyhole properties
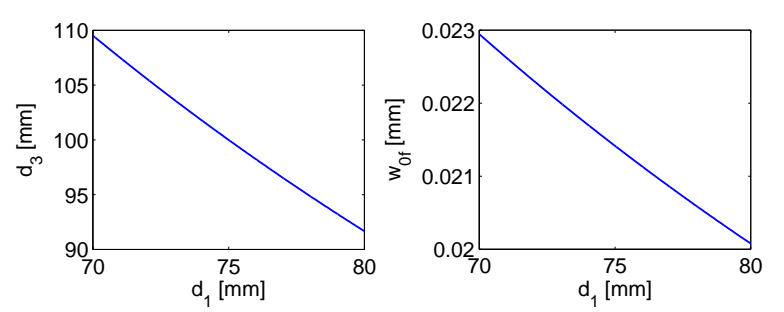

Fig. 4. Dependence of final waist radius $w_{0 \mathrm{f}}$ and its distance from the focusing lens $d_{3}$ on distance between the end of optical fiber and the collimating lens $d_{1}$

on the shape of laser beam incident on the work piece. In the following text we will give a brief mathematical description of particular phenomena involved in the model.

The laser beam incident on the work piece will be assumed to have a Gaussian intensity profile given by

$$
I(r, z)=I_{0}\left(\frac{w_{0 \mathrm{f}}}{w(z)}\right)^{2} \exp \left(-\frac{2 r^{2}}{w^{2}(z)}\right) .
$$

The value $I_{0}=2 P / \pi w_{0 \mathrm{f}}^{2}$, where $P$ is total power of the beam and $w_{0 \mathrm{f}}$ is a waist radius calculated in the previous section. The function $w(z)$ that describes the beam radius along its axis of propagation is given by

$$
w(z)=w_{0 \mathrm{f}} \sqrt{1+\left(\frac{z-z_{0}}{z_{\mathrm{r}}}\right)^{2}},
$$

where $z_{0}$ is now a waist position with respect to the surface of welded material.

The absorptivity of the welded material can be calculated as $1-R$, where $R$ is reflectivity of the molten material described by the Fresnel angle dependent model [14]

$$
R=\frac{1}{2}\left(\frac{1+(1-\varepsilon \cos \theta)^{2}}{1+(1+\varepsilon \cos \theta)^{2}}+\frac{\varepsilon^{2}-2 \varepsilon \cos \theta+2 \cos ^{2} \theta}{\varepsilon^{2}+2 \varepsilon \cos \theta+2 \cos ^{2} \theta}\right) .
$$

Here $\theta$ is an angle between incident ray and surface normal, the quantity $\varepsilon$ depends on laser type and material properties. We will accept a value 0.08 widely used for combination of $\mathrm{CO}_{2}$ laser and steel work piece [14].

The absorbed intensity represents a heat source term $Q(\mathbf{r}, t)$ in time-dependent heat equation

$$
\rho c \frac{\partial T(\mathbf{r}, t)}{\partial t}=\Lambda \nabla^{2} T(\mathbf{r}, t)+Q(\mathbf{r}, t)
$$

that determines the distribution of temperature $T(\mathbf{r}, t)$ in the material. Here all the parameters, i.e. density $\rho$, heat capacity $c$ and heat conductivity $\Lambda$ are assumed to be constant.

The multiple reflections of laser beam inside the keyhole will be considered within the frame of geometrical optics - the beam is represented by a finite set of rays. The reflection of a ray incident at the keyhole wall of surface normal vector $\mathbf{n}$ is given by the relation

$$
\mathbf{k}=\mathbf{i}+2(-\mathbf{i} \cdot \mathbf{n}) \mathbf{n},
$$

where the vectors $\mathbf{i}$ and $\mathbf{k}$ correspond to the direction of propagation of incident and reflected ray, respectively. 
Attenuation of laser beam intensity in plasma due to the inverse Bremsstrahlung is driven by Beer-Lambert's law

$$
I(z)=I(0) \exp \left(-\alpha_{i B} z\right)
$$

where $I(0)$ is incident intensity, $I(z)$ is intensity transmitted after propagation along the distance $z$ and $\alpha_{i B}$ is a plasma absorption coefficient.

One of the important keyhole characteristic, we are also interested in, is the surface temperature $T_{s}$ of the keyhole walls. This temperature depends especially on the amount of absorbed intensity $I_{a b s}$ and heat of evaporation of the welded material $L_{v}$. It can be calculated from the following two equations [15] binding the surface temperature and speed of evaporation front $v_{s}$

$$
\begin{aligned}
& I_{a b s}=v_{s} \rho\left(L_{v}+c T_{s}\right), \\
& v_{s}=v_{0} \exp \left(-\frac{M L_{v}}{k_{B} T_{s}}\right),
\end{aligned}
$$

where $v_{0}$ is a constant corresponding to the speed of sound in the welded material, $M$ is its atomic mass and $k_{B}$ is the Boltzmann constant.

\section{Numeric simulation}

The aim of the numeric simulation is to estimate the geometry of the keyhole and the surface temperature and absorbed intensity distribution on its walls on the basis of known (or estimated) parameters of the incident laser beam (geometry of beam waist and its position to material surface), the work piece material and the physical phenomena that take part in the process of the keyhole creation.

The simulation is limited to two dimensions and deals with a longitudinal cross-section of the welded material, i.e. the plane given by the axis of the incident laser beam and the axis of the beam movement over the work piece surface. The material cross-section plane is modelled as a discrete mesh, i.e. a 2D matrix representing discrete infinitesimal areas of the material. The laser beam is considered to have ideal Gaussian distribution of intensity. The plasma that fills the keyhole and that creates the plume above it is described by a plasma absorption coefficient which is assumed to be constant. Regarding the temporal scale discrete time steps are used to evaluate the course of events within the material.

The simulation starts with intact work piece heated up to the ambient temperature and it is run until the keyhole depth is stabilized at a constant value. Then the results of simulation performed for different parameters are easily cross-comparable.

The simulations were performed for mild carbon steel, beam power $2 \mathrm{~kW}$, welding speed $1 \mathrm{~m} / \mathrm{min}$ and the focusing optics of the $\mathrm{CO}_{2}$ laser welding machine. The common set of material parameters used for calculations is given in Table 1. A typical output of the numeric simulation is shown in Figure 5 - the longitudinal cross-section of the welded material with a keyhole body created and temperature distribution. The picture indicates that the layer of molten material surrounding the front wall of the keyhole is very thin. The relative motion of laser beam with respect to the work piece causes a difference between shape of the rear and the front wall of the keyhole.
Table 1. Physical properties of mild steel, plasma vapours and laser beam used in numeric simulation.

\begin{tabular}{lll}
\hline Property & Symbol & Value \\
\hline Density & $\rho$ & $7833 \mathrm{~kg} \cdot \mathrm{m}^{-3}$ \\
Heat capacity & $c$ & $650 \mathrm{~J} \cdot \mathrm{kg}^{-1} \cdot \mathrm{K}^{-1}$ \\
Heat conductivity & $\Lambda$ & $55 \mathrm{~W} \cdot \mathrm{m}^{-1} \cdot \mathrm{K}^{-1}$ \\
Melting temperature & $T_{\mathrm{m}}$ & $1500^{\circ} \mathrm{C}$ \\
Boiling temperature & $T_{\mathrm{b}}$ & $2750^{\circ} \mathrm{C}$ \\
Speed of sound & $v_{0}$ & $6000 \mathrm{~m} \cdot \mathrm{s}^{-1}$ \\
Latent heat of evaporation & $L_{\mathrm{v}}$ & $6.10^{6} \mathrm{~J} \cdot \mathrm{kg}^{-1}$ \\
Material dependent quantity & $\varepsilon$ & 0.08 \\
Plasma absorption coefficient & $\alpha_{\mathrm{iB}}$ & $320 \mathrm{~m}$ \\
Laser wavelength & $\lambda$ & $7833 \mu \mathrm{m}$ \\
\hline
\end{tabular}

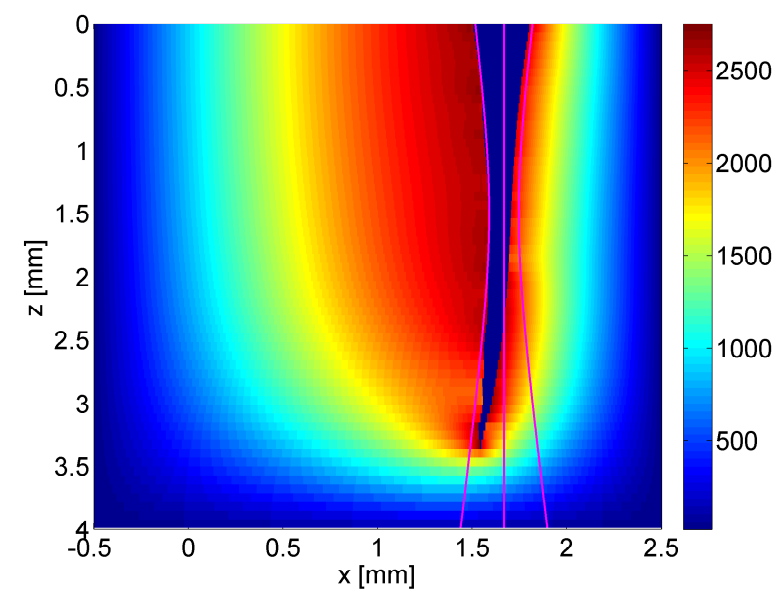

Fig. 5. Result of simulation - shape of key hole with temperature distribution $\left({ }^{\circ} \mathrm{C}\right)$ within the material around the keyhole. The laser beam indicated by its edges and axis moves to the right

The first set of results reveals a dependence of keyhole depth on waist position of the beam for different focal length of the converging lens (while the curvature of adaptive mirror is constant). The relative position of the beam waist and work piece surface was changed by vertical adjustment of the lens position. The range of waist position was from 5 millimeters below the surface up to 2 millimeters above it. For the focal length of the lens the values of 5.0" and 7.5" were chosen. The results of performed simulations are shown in Figure 6a). We see that the choice of waist position strongly affects the depth of the keyhole. For given focal length of the lens the dependence has a single maximum around which it is not symmetrical. The difference between the curves corresponding to different focal lengths lies in their maximum value and slope. The smaller focal length causes the greater maximum depth of penetration but the decrease from the maximum value is much faster.

Another set of results shows the difference between the depth of keyhole for the optical system with variable vertical position of the converging lens (while the curvature of the adaptive mirror is constant) and variable curvature of the adaptive mirror (while the position of the converging lens is fixed). It is apparent from Figure 6b) that using the adaptive optics it is possible to achieve greater keyhole depth and, moreover, it is possible to change the keyhole depth more quickly. 

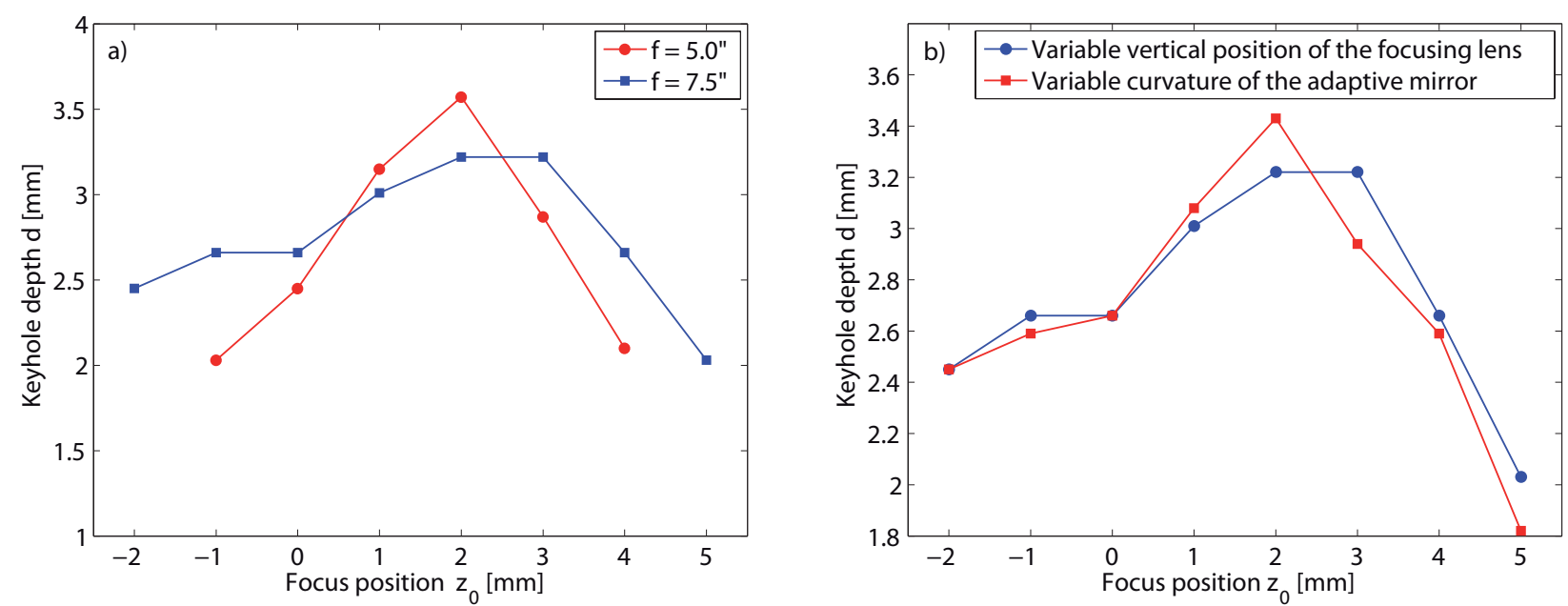

Fig. 6. a) Calculated keyhole depth $d$ depending on focus position $z_{0}$ for different focal lengths $f$ and constant curvature of adaptive mirror. b) Comparison of the keyhole depth achieved for variable vertical position of the converging lens and variable curvature of the adaptive mirror

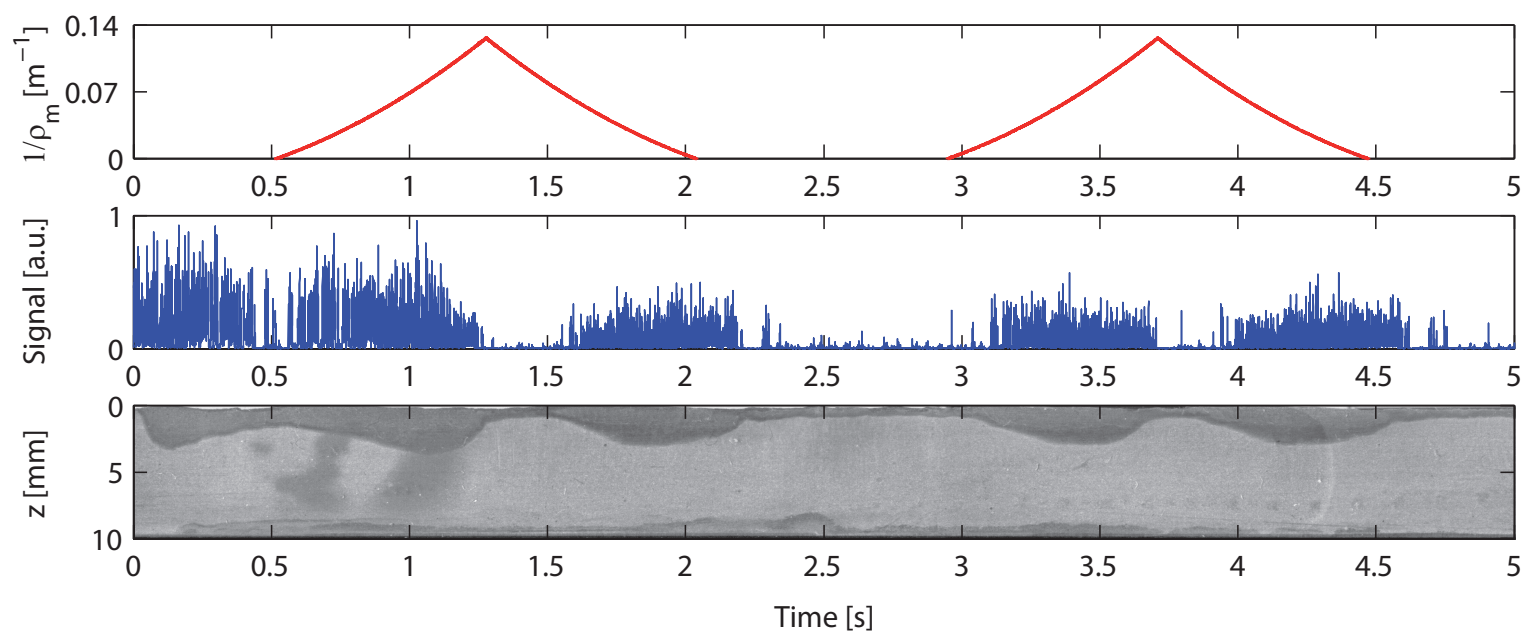

Fig. 7. Welding experiment with variable curvature of the adaptive mirror $1 / \rho_{\mathrm{m}}$

\section{Experimental verification}

To verify the results discussed above we have performed an extensive set of experiments with $\mathrm{CO}_{2}$ laser welding machine. As an example, we show on Figure 7 the evaluation of testing weld performed for the same material and welding parameters which were used in the numeric simulation, i.e. mild carbon steel, laser beam power $2 \mathrm{~kW}$ and welding speed $1 \mathrm{~m} / \mathrm{min}$, the focal length of the converging lens was $f=7.5$ ”.

The curvature of the adaptive mirror was changed along the welded distance in the range from minimum to maximum possible value (these can be found in Figure 2). Simultaneously, the welding process was monitored by photo detector sensitive on radiation emitted by plasma plume.

It is obvious from Figure 7 that the value of mirror curvature has significant impact on the weld depth. In parts of minimal weld depth the keyhole was not even created because the energy density incident on the surface of work piece was too low. These parts of shallow penetration correspond to dramatic fall in the course of signal coming from the photo detector. The evaluation of the relation between the keyhole depth and focusing parameters allowed us to conclude, that the theoretical and experimental results are in good agreement. This means that the adaptive optics is well applicable for the control of laser welding process.

\section{Conclusion}

We have presented the study of relations between keyhole characteristics and laser beam geometry given by the optical system of the $\mathrm{CO}_{2}$ and solid-state laser welding machine. For this purpose we have described the effect of different optical elements on the beam geometry after its passing through the optical system. We have also evolved a simple physical model of keyhole creation and implemented the numeric simulation of this process. Thanks to the numeric simulation we were able to study the dependence of keyhole depth, absorbed energy distribution and surface temperature distribution on laser beam geometry. Even with such a simple physical model of keyhole creation we have shown that keyhole characteristics strongly depend on the laser beam shape. The results obtained from numeric simulations were in good qualitatively agreement with results of performed experiments. 


\section{Acknowledgements}

This work was supported by the Ministry of Industry and Trade of the Czech Republic, project number 2A-3TP1/113 and was partially supported by European Commission and Ministry of Education, Youth, and Sports of the Czech Republic (project No. CZ.1.05/2.1.00/01.0017).

\section{References}

1. C.Y. Ho, M.Y. Wen, J. Mater. Process. Tech. 145, 303$310(2003)$

2. X. Jin, L. Li, Y. Zhang, J. Phys. D Appl. Phys. 35, 2304$2310(2002)$

3. X. Jin, P. Berger, T. Graf, J. Phys. D Appl. Phys. 39, 4703-4712 (2006)

4. X. Jin, P. Berger, T. Graf, J. Phys. D Appl. Phys. 30, 3216-3222, (1997)
5. J. Dowden, P. Kapadia, J. Phys. D Appl. Phys. 28, 22522261 (1995)

6. M.R. Frewin, D.A. Scott, Weld. J., 78, 15-22 (1999)

7. A. Kaplan, J. Phys. D Appl. Phys. 27, 1805-1814 (1994)

8. J.H. Cho, S.J. Na, J. Phys. D Appl. Phys. 39, 5372-5378 (2006)

9. H. Ki, P.S. Mohanty, J. Mazumder, J. Phys. D Appl. Phys. 34, 364-372 (2001)

10. H. Ki, P.S. Mohanty, J. Mazumder, Metall. Mater. Trans. A 33A, 1817-1830 (2002)

11. H. Ki, P.S. Mohanty, J. Mazumder, Metall. Mater. Trans. A 33A, 1831-1842 (2002)

12. A. Otto, Phys. Proc. 12, 11-22 (2011)

13. O. Svelto (2009) Principles of Lasers, (Springer, 2009).

14. W. Schulz, G. Simon, H.M. Urbassek, I. Decker, J. Phys. D Appl. Phys. 20, 481-488 (1987)

15. S.I. Anisimov, V.A. Khokhlov, Instabilities in Lasermatter interaction, (CRC Press, 1995) 